\section{AB006. Postintubation tracheal stenosis-Massachusetts General Hospital results and outcomes}

\section{Douglas J. Mathisen}

Massachusetts General Hospital, Harvard Medical School, Boston, MA, USA

Correspondence to: Douglas J. Mathisen, MD. Thoracic Surgeon, Massachusetts General Hospital, Boston, MA, USA; Hermes C. Grillo Distinguished Professor of Thoracic Surgery, Harvard Medical School, Boston, MA, USA. Email: dmathisen@mgh.harvard.edu.

Abstract: Postintubation tracheal stenosis (PITS) results from injury from cuffs or from a tracheostomy. Despite many advances in tube design, postoperative care, anesthesia and surgery, PITS continues to present a challenge to this day. Cuff pressure should be adjusted and deflated periodically. Traction on tracheostomy tubes should be avoided. Anyone who has been intubated or had a tracheostomy in the preceding months that presents with shortness of breath should be considered to have tracheal stenosis. It is imperative to secure the airway before further evaluation. If one is confident that the airway is stable, simple chest X-ray can sometimes show an area of narrowing, but it oftentimes requires a computerized axial tomogram of the chest to identify the stenosis, its location, and extent and how narrow the stenosis is. Simple measures in the emergency ward include humidified oxygen, heliox if there is significant shortness of breath, and evaluation in the operating room under general anesthesia to dilate the lesion initially. The goal initially is to secure the airway. Dilation can be done under direct vision with the flexible bronchoscope and a tracheal balloon dilator. Alternatively, rigid bronchoscopy can be used to identify the lesion and gradually dilate with a series of rigid bronchoscope starting with a \#4 pediatric bronchoscope. All associated medical problems can then be resolved and an elective repair contemplated. There is rarely a need for an emergency tracheostomy. We recently published our last 24-year experience with PITS comprising 392 patients. There were an equal number of men and women, and average age was 46 years ( 3 to 84 years). The most frequent comorbidities were obesity, diabetes, and cardiac disease. The etiology of the stenosis when it could be determined was stomal stenosis in 169 and a cuff stenosis in 143 . The mean and median length of stenosis was $3 \mathrm{~cm}$. Ninety-one of the patients had a laryngotracheal stenosis and 301 a stenosis that involved only the trachea. A prior tracheostomy, at some point had been performed in 275 . Over $95 \%$ were elective operations. The standard surgical repair consisted of an open anastomosis performed with interrupted 4-0 Vicryl sutures and 2-0 Vicryl stay sutures. This anastomotic technique was developed by Grillo over 60 years ago. A cervical approach was used in $94 \%$ of patients. A laryngeal release was utilized in only 15 of the 392 and over $94 \%$ of patients had vascularized tissue covering the anastomosis. There were three deaths associated with surgery ( $0.8 \%$ mortality). Complications occurred in 126 of the 392 patients (32\%), but 267 had no postoperative complications. The most common complication was granulation tissue related to the use of permanent suture material. Use of absorbable sutures has markedly decreased this problem. Postoperative edema was present in 56 patients predominantly in laryngotracheal resections. Fifteen patients or $4 \%$ developed wound infections. Nineteen patients required reintubation following surgery. Dehiscence and/or necrosis occurred in 14 and 12 patients respectively. Voice change related to recurrent nerve trauma was present in $4 \%$ of patients. Three hundred and one patients had tracheal resection for stenosis confined to the trachea with a $96 \%$ good or satisfactory outcome. Only $3.3 \%$ of these patients failed. Laryngotracheal resection and reconstruction had a good or satisfactory outcome in $85 \%$. Forty-five patients had failed a prior resection and reconstruction and had a $91 \%$ good or satisfactory result. Those patients having calcified airways had a good or satisfactory outcome in $87 \%$. Over 70 patients had been on steroids. The 35 patients were weaned to a median dose of $5 \mathrm{mg}$ of prednisone. Thirty-five patients on steroids had a $91 \%$ good or satisfactory outcome. There were only 12 patients who had resections of greater than $5 \mathrm{~cm}$ with an $89 \%$ good or satisfactory outcome. One hundred and twenty-three patients were diabetic and had an $85 \%$ good or satisfactory outcome. All but 31 patients in this series had some type of prior intervention. Two hundred and seventyfive had a preoperative tracheostomy. One hundred and fifty-two of these patients had their tracheostomy removed prior to surgery and had a near normal outcome with $96 \%$ success. If a tracheostomy was present at the time of surgery, this conferred a worse prognosis with an $86 \%$ good 
or satisfactory outcome. If there was concern at the time of surgery and a protecting tracheostomy was placed, as was the case in 22 patients, a good or satisfactory outcome was achieved in only $74 \%$. If the patient initially left the operating room without a tracheostomy, but developed a problem that required a tracheostomy, the outcome in that group of patients fell to only a $38 \%$ good or satisfactory outcome with $54 \%$ patients failing. T-tubes $(\mathrm{n}=61)$ or stents $(n=44)$ also conferred a less favorable outcome with only $90 \%$ good or satisfactory outcome. Multiple laser dilations did not have an impact on outcome. The conclusions from this study identified factors that influence the outcome following tracheal resection and reconstruction for PITS. Laryngotracheal stenosis, a tracheostomy present at the time of surgery, the requirement for a postoperative tracheostomy, T-tubes or stents preoperatively and major postoperative complications all influence the outcome of patients having tracheal resection and reconstruction. The MGH experience in the most recent report combined with Dr. Grillo's seminal report spans nearly 55 years and encompasses 900 patients. The two series are similar in many ways. The main differences between the two series was the increased mortality of $2.4 \%$ in the older series compared to the current series of $0.8 \%$. Over two thirds of the patients in the current series had some type of prior procedure with tracheostomy being the most common compared to only $8 \%$ in the original series. Fewer patients in the current series had a release maneuver (4\%) compared to $10 \%$ in the Grillo series. Eleven percent of patients in the current series had a stent preoperatively, but none in the original series. Both series had an incidence of complications of roughly $33 \%$. The overall results in both series was a good or satisfactory outcome in $94 \%$. Laryngotracheal stenosis was more common in the contemporary series with 91 patients presenting with this problem compared to 62 in the prior series. Outcomes were similar with $90 \%$ good or satisfactory outcome in the first series compared to $85 \%$ in the present series. Re-operations were carefully selected with outcomes in the current series of nearly $90 \%$ of good and satisfactory compared to $87 \%$ in the Grillo series. These results demonstrate that PITS continues to be a serious problem. The best results are achieved when paying strict attention to detail and patient preparation. Despite the differences in the two series, similarly good results can be achieved with careful attention to detail, precise surgical technique, dedicated postoperative care and understanding the principles of tracheal surgery.

Keywords: Trachea; stenosis; complications; results

doi: 10.21037/shc.2019.AB006

Cite this abstract as: Mathisen DJ. Postintubation tracheal stenosis-Massachusetts General Hospital results and outcomes. Shanghai Chest 2019;3:AB006. 